\title{
Entendiendo la arquitectura sostenible como un ente generado esencialmente a partir de su entorno
}

\author{
Understanding sustainable architecture as an entity \\ essentially generated from its environment
}

\section{Autor:}

Tomás Galindo

Universidad de Cuenca

Recibido:

15 de febrero 2012

Aceptado:

15 de Marzo 2012

\section{Resumen}

Este artículo sugiere una aproximación sui generis a la concepción de anteproyecto arquitectónico sostenible, en donde la respuesta que la arquitectura proporciona a la problemática establecida está definida exclusivamente a partir de los resultados tangibles que proporciona el análisis de una serie de condicionantes específicos, determinados por el entorno o contexto arquitectónico, así como por los parámetros funcionales implícitos dentro del tema de proyecto. Evidentemente, cabe resaltar que la temática de este artículo se ve reflejada en su contenido por medio de una descripción y apreciación personal de su autor sobre el método evidenciable a partir de la lectura del documento de tesis de grado: "Centro de Investigación Científica con fines turísticos \& Museo de Meteorología en la isla San Cristóbal, provincia de Galápagos", documento citado en la bibliografía de este artículo.

Palabras clave: Arquitectura, sostenibilidad, anteproyecto, Ecuador.

\begin{abstract}
This article suggests an approach sui generis to the conception of sustainable architecture preliminary project, where the response provided by the architecture to the problem set is defined exclusively from the tangible results that provides analysis of a series of specific factors, determined by the environment or architectural context, as well as by the implicit functional parameters within the subject of project. Obviously, should be noted that the subject of this article is reflected in its contents through a description and personal appreciation of its author on the method noticeable from the reading of the thesis paper: "Scientific research center for tourism \& Museum of Meteorology in San Cristóbal Island, Galapagos province", document cited in the bibliography of this article.
\end{abstract}

Keywords: Architecture, sustainability, preliminary draft, Ecuador. 


\section{Introducción:}

¿Cuál es el punto de partida que un anteproyecto arquitectónico debe tomar si se considera que éste se emplazará en un contexto de características naturales y socioculturales que se encuentran en contraposición y conflicto? ¿Cómo se debe proceder para conciliar, por medio de la arquitectura, necesidades de orden natural por un lado, y de orden social por el otro? Considerando además, que dentro de tal contexto lo social depende de lo natural, mas sin embargo que lo natural independientemente de lo social, se encaminaría fluidamente en aras de su propia preservación. Tras entender este contexto general, inevitablemente surge a la luz otra interrogante: ¿Cómo es que la arquitectura, siendo un ente artificial, y por lo tanto un mero representante de la parte sociocultural, podría solucionar un conflicto en donde la contraparte natural simplemente no tiene un claro defensor de sus intereses? ¿O es que tal vez, sí lo tiene?...

Éste es el complejo meollo a partir del cual nace el diseño de cualquier arquitectura contemporánea, puesto que se trata de una problemática cuyo conflicto se generaliza cada vez más en el mundo actual. Meollo que no obstante, otorga a la vez el camino que permite encontrar las respuestas necesarias para el planteamiento formal del anteproyecto, en conjunción con las necesidades funcionales contempladas por el tema del programa arquitectónico.

De esta manera, gracias al método que se expondrá en este artículo y que se deduce a partir de las problemáticas previamente planteadas, el proyecto arquitectónico literalmente se puede generar como un "ente" "espontáneo" " de principio a fin, restando para los proyectistas del mismo tan sólo el papel de orquestes de aquello que ya se encuentre implícita y explícitamente establecido dentro del contexto a ser intervenido. Así, el siguiente esquema resume tal método, en donde el condicionante de uso (función) se restringe a los condicionantes de forma puesto que éstos últimos obedecen a características irrepetibles que requieren de respuestas puntuales, mientras que la función responde a usos de características universales.

\section{Método propuesto:}

Generalmente en un diseño arquitectónico se comienza por analizar el programa funcional, tomando este análisis como columna vertebral del proyecto. Sin embargo, este proceso deja de lado más del $80 \%$ de los condicionantes imprescindibles dentro de un proyecto, sobre todo si ambiciona ser sostenible. El tema funcional constituye un aspecto pre establecido en la propia temática del proyecto, además de ser un aspecto universal que funciona a manera de "plantilla" para cualquier caso de estudio. La universalidad de este aspecto lo convierte simplemente en un punto más a considerar dentro del diseño. Por lo tanto, a fin de establecer la verdadera columna vertebral del proyecto, se debe analizar primero los factores más característicos que se encuentran ya en el lugar donde se emplazará el futuro edificio, pues para éstos deben ser desarrolladas respuestas específicas que carecen de fórmulas generales.

Se parte entonces de los cuatro aspectos señalados con anterioridad, tres contextuales (predio o solar, entorno, sostenibilidad) y uno pre establecido (funcionalidad). El resultado de estos condicionantes generará el diseño arquitectónico final.

\section{A. Predio o Solar:}

Aspectos que definen el tema "predio o solar":

- Sitio geográfico: proporcionará datos generales sobre el clima específico de la zona. No implica lo mismo proyectar para la zona ecuatorial, que para una de mayor o menor altitud, o para un piso climático u otro, etc. Estos parámetros climático-arquitectónicos se los clasifica en tres: medioambientales, corporales y de proyecto. El manejo de los tres define el confort térmico al interior de un edificio y que se define como "el punto en el que el hombre gasta la energía mínima para adaptarse a su entorno" ${ }^{4}$.

- Composición geográfica y topográfica: la topografía por un lado, evidentemente permite conocer la morfología real de la superficie del sitio, pero sobre todo en este punto se establece desde ya la mejor volumetría posible a ser implantada en el terreno, y que mejor aproveche su forma. La geología por otro lado, señala las claves sobre el tipo de cimentación que se deberá utilizar, por lo que el proyecto queda definido ya desde este análisis en cuanto a sus aspectos volumétricos.

- Aspectos arquitectónico-urbanos: el estudio de estos aspectos permite rescatar valores arquitectónicos que se puedan emplear en el proyecto. En el caso de no existir tales valores, se registran los errores arquitectónicos que no se desean repetir en el proyecto y de esta manera se propone una solución o antítesis de los errores observados.

- Aspectos de paisaje y cromática: contempla todo elemento adyacente en torno al terreno. Sin embargo se deben rescatar exclusivamente los valores cromáticos que mejoren la calidad del paisaje general de la zona a intervenir. Por ejemplo, en el caso de un sitio rodeado de abundante vege- 

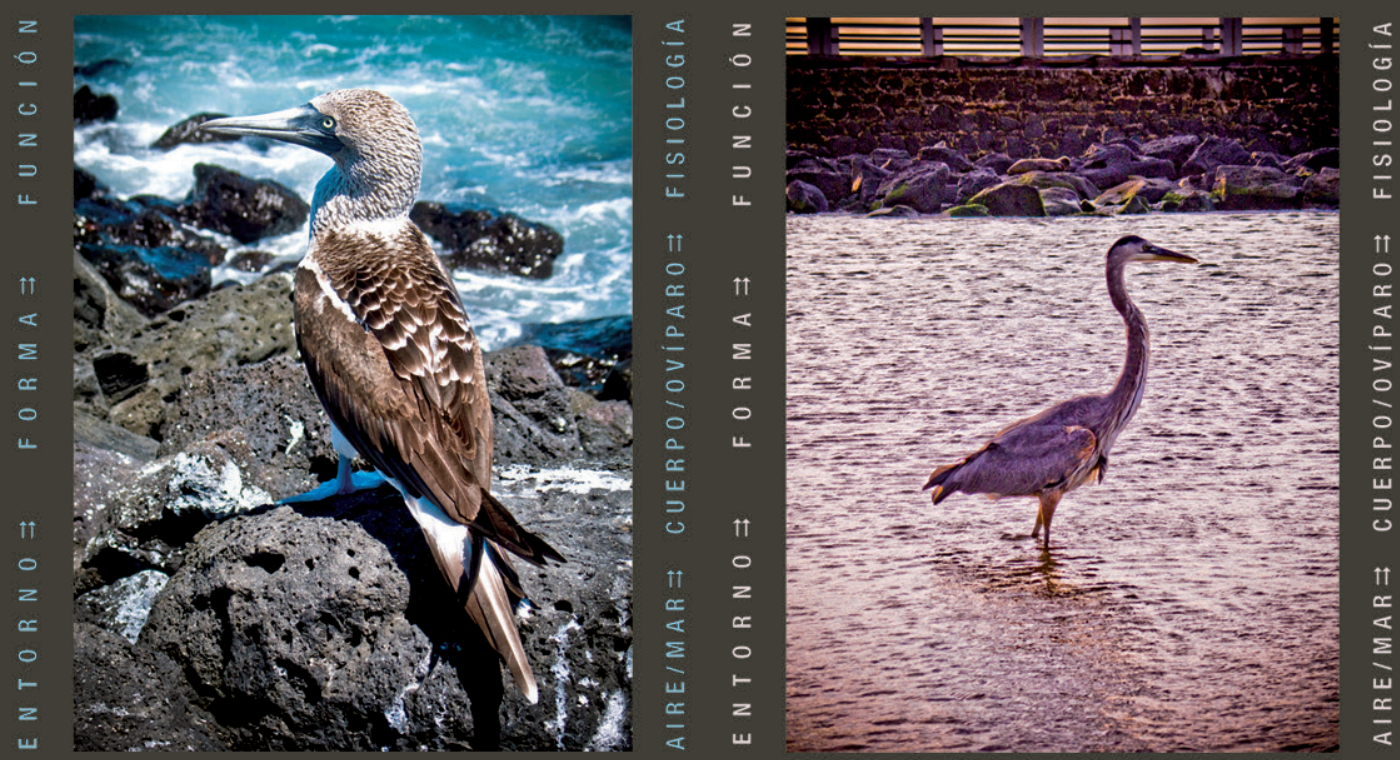

ENTORNO $\Rightarrow \quad \mathrm{FORMA \rightrightarrows} \quad \mathrm{FUNCION}$

ENTORNO $\Rightarrow \quad \mathrm{FORMA} \rightrightarrows \quad \mathrm{FUNCION}$
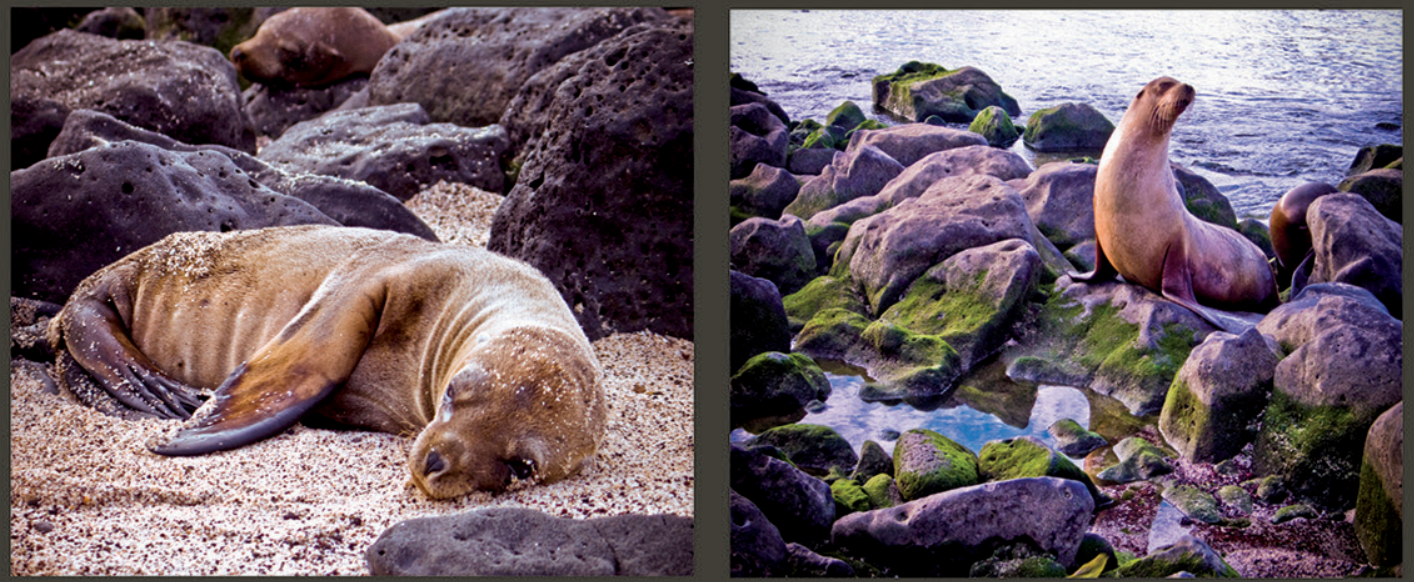

ROCAS/MAR $\Rightarrow$ CUERPO/MAMIFERO $\Rightarrow$ FISIOLOGIA

ROCAS/MAR $\rightrightarrows$ CUERPO/MAMIFERO $\Rightarrow$ FISIOLOGIA

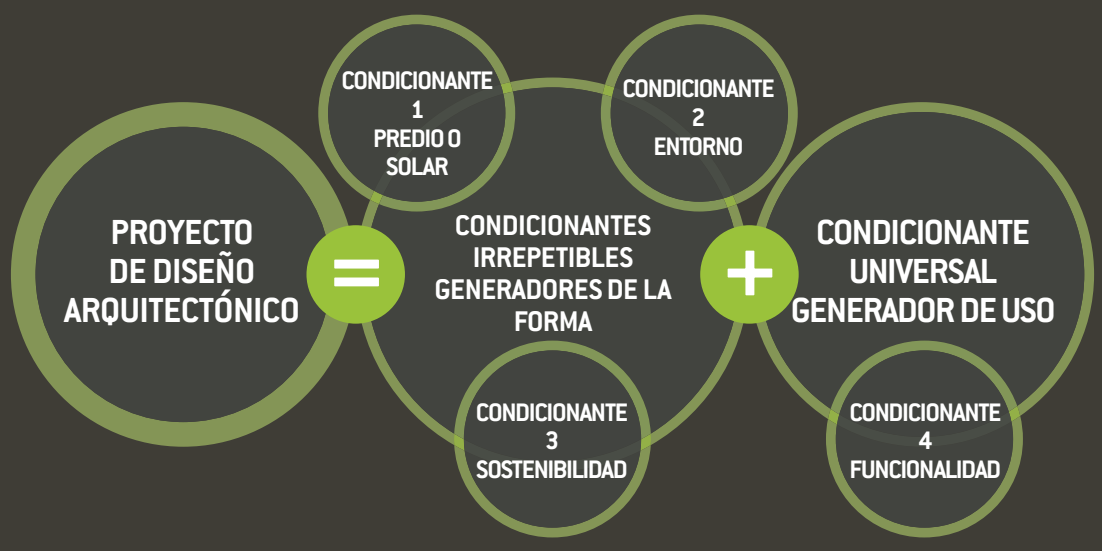


tación nativa, es imprescindible analizar principalmente la cromática del entorno natural pues ésta representa el valor de la zona como posible patrimonio natural, y que podría estar siendo invadida por edificaciones aleatorias que destruyen su paisaje endémico. Por ello en tales casos se deberá hacer caso omiso de la cromática de elementos artificiales, rescatando sólo aquella del entorno nativo natural a fin de contrarrestar la destrucción del paisaje.

\section{B. Entorno:}

Los aspectos que definen el "entorno" son específicos del contexto inmediato de cada terreno a intervenir, y son:

- Aspectos climáticos: evidentemente se analizarán las precipitaciones, el soleamiento, los vientos, las temperaturas, etc. Cada uno de estos aspectos climáticos proporcionará diferentes claves específicas a ser utilizadas arquitectónicamente, las mismas que serán conjugadas con la volumetría general obtenida anteriormente por el tema geográfico y topográfico. Por ejemplo, este análisis proporciona criterios básicos sobre aberturas o vanos, orientación de las caras del edificio, elementos generadores de sombra, ubicación de espacios abiertos, empleo de aislantes y amortiguadores térmicos como la vegetación y el agua, orientación de las caras y formas del edificio para la captación del viento y su fuerza cinética, disposición de elementos captadores de agua, disposición de zonas ideales de captación solar (energías renovables), etcétera.

- Aspectos naturales: se pueden subdividir en flora y fauna. Se debe considerar además que para efectos arquitectónicos, este tema no es importante desde un punto de vista puramente botánico o zoológico, sino como fuente de datos a ser empleados en el proyecto. Por ejemplo, a partir de este tema se pueden obtener datos en cuanto a las texturas y cromáticas de los elementos naturales y cómo estos aspectos benefician a su entorno nativo inmediato, lo cual básicamente se refleja en la conservación y la armonía. Estos aspectos relacionados con el mimetismo, se pueden emplear en el proyecto con el fin de conseguir una armonía con el entorno y evitar tornar el proyecto en un elemento intruso e irrespetuoso con el sitio en el cual se intervenga, sobre todo si se trata de un medio ambiente exclusivamente natural. A fin de lograr tales objetivos, se deben emplear materiales locales y resaltar sus texturas, además de una vegetación preferentemente nativa en- tendida como material constructivo, mas no como un elemento ornamental, pues ésta contiene características termodinámicas muy beneficiosas 5 . Además las formas arquitectónicas empleadas, al responder a condicionantes medioambientales específicos, tienden a ser inevitablemente formas características que podrían entenderse como "naturales u orgánicas" pues nacen de un proceso que da una respuesta que se adapta a un ciclo natura y que por lo tanto se tornará eventualmente en parte de esa naturaleza.

- Antecedentes socio-arquitectónicos: en este punto se realiza un "background" histórico del porqué de la arquitectura existente en el entorno y sus causas, con el fin de rescatar valores arquitectónicos o de entender los errores efectuados, para comprender sus orígenes e intentar dar respuesta a estas situaciones por medio de arquitectura. Esto, básicamente estableciendo la nueva obra como ejemplo a seguir en el medio que se intervenga.

\section{Sostenibilidad}

No se puede desarrollar un proyecto sostenible si no se conocen primero los siguientes puntos esenciales:

Criterios generales: la noción de desarrollo sostenible es un concepto utilizado desde la antigüedad por muchas de las grandes civilizaciones humanas, como la Inca por ejemplo. Sin embargo, en nuestra era la Comisión Brundtland (1987) definió el desarrollo sostenible como "aquél que satisface las necesidades del presente sin comprometer la capacidad de las generaciones futuras de satisfacer las suyas propias". ${ }^{6}$ A partir de esta definición, el tema se ha desarrollado en varias áreas principalmente en la construcción. Es precisamente en esta área en donde se consume "el 50\% de los recursos mundiales" 7 , siendo la sostenibilidad no una opción sino una obligación en la concepción de la arquitectura contemporánea.

- Energía limpia sostenible: debe aclararse que éstas deben ser limpias o libres de emisiones tóxicas. Por ello, para la arquitectura son básicamente la solar, la eólica y la hídrica. Su uso y aplicación dependerán de los factores climáticos previamente analizados en la sección de "entorno", y según este criterio se dispondrán de espacios y formas específicas en el proyecto que faciliten el empleo de las mismas.

Aspectos constructivos: Finalmente, es imprescindible entender el impacto que los materiales de construcción provocarán en el medioambiente, existiendo métodos de control y monitoreo específicos. No obstante lo esencial para poder catalogar un material constructivo como sostenible es aplicando el siguiente criterio: "aprovisionamiento local de los materiales pesados", (pétreos, maderas, agregados finos y gruesos, etc.) ya que la contaminación por su transporte y extracción se disminuye y el impacto ambiental es más manejable y de mucho menor escala, destinando zonas específicas de explotación y restaurando con más facilidad los ecosistemas explotados en el largo plazo. "Aprovisionamiento global de 

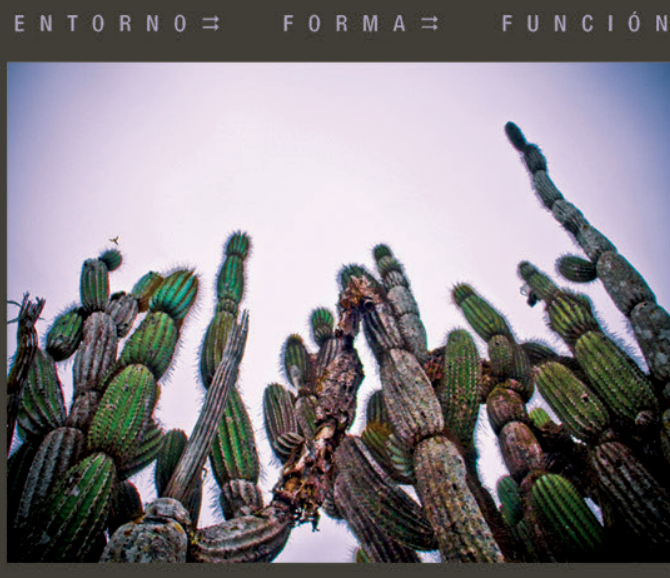

TIERRA $\Rightarrow \quad$ CUERPO/VEGETAL $\Rightarrow \quad \mathrm{FISIOLOGIA}$

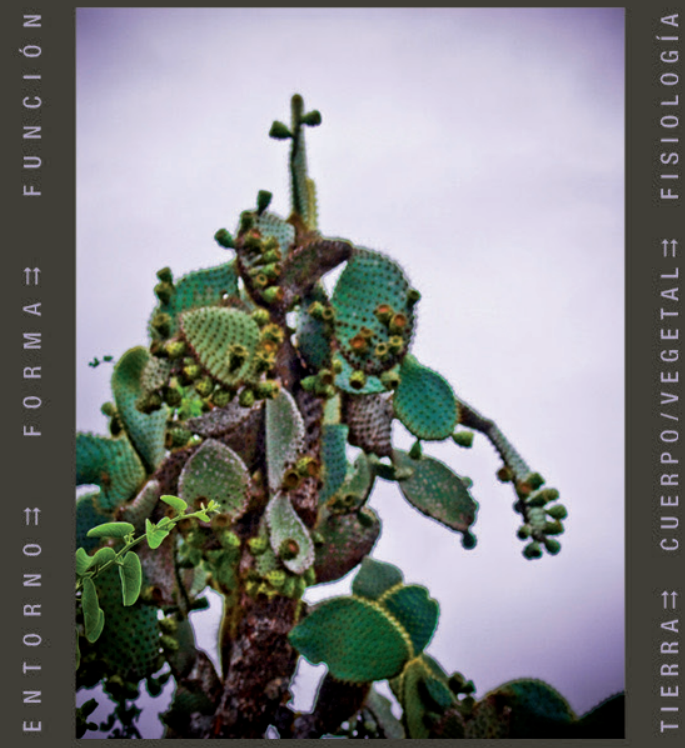

A fin de obtener una idea arquitectónica más gráfica del proceso expuesto en este artículo, el lector puede referirse al ejemplo similar planteado en el documento de tesis de grado citado como bibliografía de este artículo.

\section{Conclusiones:}

Una vez que se ha establecido un eje o columna vertebral específica para el proyecto que se desea plasmar, y que se cuenta con todos los elementos necesarios para definir la morfología que responda de la manera más eficiente a los condicionantes específicos dictados por el entorno a intervenir, es momento de analizar la función planteada por el propio tema de proyecto.

Además de esto, se necesita de una guía que otorgue las pistas de cómo proceder arquitectónicamente concerniendo a los espacios requeridos y dejando de lado por el instante lo deducido anteriormente a partir de los análisis de predio, entorno y sostenibilidad. Por esta razón es necesario realizar un breve análisis de obras arquitectónicas renombradas que cumplan con criterios de eficiencia y de responsabilidad con su entorno social, cultural, natural y económico. Estos análisis tienen como objetivo extraer criterios básicos de cómo proceder funcionalmente en base a buenas experiencias adquiridas por terceros.

\section{E. El diseño de anteproyecto}

Es tiempo de ejecutar lo recopilado, analizado y aprendido. En este punto conviene articular los datos procesados ya en términos arquitectónicos, de manera que den forma al proyecto como mejor satisfaga las necesidades tanto socioculturales como naturales expuestas al inicio. No queda, sino realizar bien la suma de los datos obtenidos y así llegar al resultado correcto, satisfaciendo de este modo la problemática inicial representada por el conflicto entre intereses contrapuestos.
La deducción de este método de diseño nació de una problemática específica, la cual se considera en mayor o menor medida como denominador común de todo planteamiento arquitectónico actual. De este modo, se replantean las inquietudes señaladas inicialmente y se responde a cada una de ellas:

- ¿Cuál es el punto de partida que un anteproyecto arquitectónico debe tomar si se considera que éste se emplazará en un contexto de característi naturales y socioculturales que se encuentran en contraposición y conflicto? El punto de partida es el análisis sistemático de los elementos que conforman el entorno o contexto inmediato, y éstos conciernen caracteres de predio o solar, entorno y sostenibilidad, además de la invariable de funcionalidad.

- ¿Cómo se debe proceder para conciliar, por medio de la arquitectura, necesidades de orden natural por un lado, y de orden social por el otro? Traduciendo los datos obtenidos de los elementos señalados en datos arquitectónicos aplicables al caso de estudio. 
- Considerando además, que dentro de tal contexto lo social depende de lo natural, mas sin embargo que lo natural independientemente de lo social, se encaminaría fluidamente en aras de su propia preservación. La naturaleza ha sido puesta al servicio del invaluable ser humano para que éste la utilice inteligentemente en pro de su bienestar común. Cuestionar tal indudable atributo de la humanidad sobre la naturaleza sólo puede llevarnos a una auto-denigración que nos etiqueta de simples "animales" dentro de la biósfera, sin olvidar la irracionalidad y perversión de la moral e inteligencia que tal autoconcepción peyorativa involucra, marcando el camino hacia una degradación social y humana que para muchos resulta cada vez más evidente en el mundo contemporáneo. Por esta razón, tan sólo la aplicación de nuestra inteligencia humana privilegiada por el discernimiento de lo que destruye o edifica, podrá eventualmente salvarnos de nuestro mal uso de los recursos naturales puestos a nuestra disposición por quien lo ha creado todo. Por ello, conceptos como la sostenibilidad permiten vivir en armonía con la naturaleza a la vez que se genera prosperidad, pero sin que esto involucre sobreponer la bandera de "conservacionismo natural" sobre la indiscutible valía humana de las cuantiosas masas demográficas que pueblan actualmente el planeta. La arquitectura por ende, al contrario de lo que sugiere el planteamiento que abre este inciso, debe proyectarse primordialmente para asegurar el bienestar humano. Así, y solamente como recurso de tal objetivo, "la sostenibilidad y la preservación natural" se pueden considerar como excelentes opciones.

- ¿Cómo es que la arquitectura, siendo un ente artificial, y por lo tanto un mero representante de la parte sociocultural, podría solucionar un conflicto en donde la contraparte natural simplemente no tiene un claro defensor de sus intereses? Porque la arquitectura, tras el análisis y método aquí planteado, se conformará de igual manera por datos conferidos por ambas contrapartes involucradas, creando un diseño que inevitablemente responderá a ambas necesidades.

- ¿ ¿O es que tal vez, sí lo tiene? La naturaleza tiene un representante establecido por condicionantes existentes explícita e implícitamente en la zona a intervenir, los cuales son de características únicas e irrepetibles para cada caso específico, además de deducibles a partir del método aquí planteado.

Se entenderá finalmente que en la arquitectura se puede aplicar un método de diseño sostenible como el aquí expuesto, mas nunca fórmulas de diseño que generen respuestas predeterminadas, pues esporádicamente los casos de intervención arquitectónica podrán llegar a asemejarse pero con certeza jamás se repetirán a fidelidad, conformándose la mayoría de tales casos por características extremadamente particulares. En base a estos fundamentos, la lógica e inevitable conclusión a la cual lleva el procedimiento planteado en este artículo permite decretar como base primaria de toda arquitectura sostenible que: "la función se acopla a la forma, pues la forma óptima siempre será la respuesta directa a un entorno particular y específico".

\section{Notas}

1 Autores de la tesis: Tomás Galindo Pazán, José Pesántez Pesántez. Director de la tesis: Arq. Honorato Carvallo Cordero.

2 La primera definición de la palabra "ente" según el www.wordreference.com, es: "Lo que es, existe o puede existir". De esta manera, se señala como ente arquitectónico a un elemento que potencialmente puede existir, llegando efectivamente a manifestarse. Se ilustra así en esta palabra, el proceso lógico que define una obra arquitectónica y que va desde su concepción efímera, hasta su materialización formal.

3 La segunda definición de la palabra "espontáneo" según el www.wordreference.com, es: "Que se produce por sí solo, sin agentes externos que lo provoquen". Para fines del presente artículo, el uso de esta definición señala como "agentes externos" a todo aquello que no sea dictado por los condicionantes propios del entorno en el cual se emplazará el proyecto arquitectónico, aparte de las demandas funcionales. Se enfatiza así, la característica principal del anteproyecto final obtenido por el método planteado en este artículo, y que es su generación exclusiva a partir de los elementos que se encuentran en su propio entorno. A partir de la definición citada queda entendido además, que al ser los proyectistas del diseño arquitectónico parte imprescindible de aquello "que se produce", éstos quedan incluidos dentro del "por sí solo" de la definición.

4 SANMIGUEL, Sandra, (versión castellana). “Un Vitruvio Ecológico, principios y práctica del proyecto arquitectónico sostenible". Editorial Gustavo Gili. Barcelona, 2010. Pág. 38.

5 "La vegetación integrada en los edificios". Internet: www.terra.org, acceso: 28 de abril de 2012.

6/9 EDWARDS, Brian. "Guía básica de la Sostenibilidad". Editorial Gustavo Gili. Barcelona, 2008. Páginas: 20, 3, 122 y 123 respectivamente. 


\section{Imágenes}

Fotografías y gráfico: Arq. Tomás Galindo.

\section{Bibliografía}

GALINDO, Tomás; PESÁNTEZ, José. "Centro de Investigación Científica con fines turísticos \& Museo de Meteorología en la isla San Cristóbal, provincia de Galápagos". Facultad de Arquitectura y Urbanismo, Universidad de Cuenca. Tesis previa a la obtención del título de Arquitecto. Cuenca, 2011. 